\title{
APPROXIMATION METHODS FOR THE SOLUTIONS OF IMPULSE DIFFERENTIAL EQUATIONS
}

\author{
A. GEORGIEVA AND S. KOSTADINOV
}

\begin{abstract}
In the present paper approximations for the solution of impulse differential equations by solutions of an appropriately constructed ordinary differential equations are found.
\end{abstract}

\section{Introduction}

The impulse differential equations are adequate models of processes which are undergoing salutatory changes in their evolutionary development in the form of impulses. These kinds of processes are commonly met in many branches of physics, chemistry, robotics, biotechnology etc. The quantitative theory of these equations has been intensively developing in the last 5 years (see [1]-[4]). In the present paper for a given impulse equation we construct an ordinary equation so that the solutions of these equations are "near" in various senses.

\section{Statement of the Problem}

We consider the following impulse equation

$$
\begin{aligned}
\frac{d x}{d t} & =f(t, x) \quad\left(t \neq t_{n}\right) \\
x\left(t_{n}^{+}\right) & =Q_{n} x\left(t_{n}\right) \quad\left(t=t_{n}\right),
\end{aligned}
$$

where $F(t, x): \mathbb{R}_{+} \times \mathbb{R} \rightarrow \mathbb{R}\left(\mathbb{R}_{+}=[0, \infty)\right), Q_{n}: \mathbb{R} \rightarrow \mathbb{R}$. The points of jump $t_{n}$ satisfy the following conditions $0=t_{0}<t_{n}<t_{n+1}(n=1,2, \cdots), \lim _{n \rightarrow \infty} t_{n}=\infty$.

The process simulated by (1), (2) evolves as follows: the point $P(t)=(t, x(t))$ which maps the trajectory having left the starting point $\left(t_{0}, x_{0}\right)$, moves along the cruve $\{t, x(t)\}$ determined by the solution of the ordinary differential equation

$$
\frac{d x}{d t}=F(t, x)
$$

Received December 15, 1997; revised January 12, 1999.

1991 Mathematics Subject Classification. 34A37, 45L05

$K e y$ words and phrases. One-sided Hausdorff metric, $L_{p}$-approximation. 
with initial value $x_{0}$ at point $t_{0}$. The motion along this curve goes on until the point $t_{1}>t_{0}$ at which the point $(t, x(t))$ meets the hyperplane $t_{1}$. At the moment $t=t_{1}$ under the action of the impulse operator $Q_{1}$ a transition by a jump is performed from the state $\left(t_{1}, x\left(t_{1}\right)\right)$ into the state $\left(t_{1}, x\left(t_{1}^{+}\right)\right)$. Further on, for $t \in\left(t_{1}, t_{2}\right)$ the mapping point $P(t)$ moves along the curve $\{t, x(t)\}$, where $x(t)$ is a solution of equation (3) with initail valued $x\left(t_{1}^{+}\right)$. At the moment $t_{2}$ new transition by a jump is carried out from the state $\left(t_{2}, x\left(t_{2}\right)\right)$ into the state $\left(t_{2}, x\left(t_{2}^{+}\right)\right)$, etc. The motion on the left of the starting point is carried out similarly.

Definition 1. The function $\varphi(t)$ is said to be a solution of (1)-(2) if for $t \neq t_{n}(n=$ $1,2, \cdots)$ it satisfies the ordinary differential equation (1) and for $t=t_{n}$ the condition of jump (2).

Further we assume that the solutions $x$ of the impulse equation are continuous on the left.

We consider the Cauchy problem for (1), (2)

$$
x\left(t_{0}\right)=x_{0}
$$

It is not hard to check, that the impulse Cauchy problem (1), (2), (4) has for any $x_{0} \in \mathbb{R}$ a unique solution off the ordinary Cauchy problem (1), (4) has for any $x_{0} \in \mathbb{R}$ a unique solution.

Lemma 1. The solution of the problem (1), (2), (4) for $t \in\left(t_{n}, t_{n+1}\right]$ is given by the formula

$$
x(t)=Q_{n} x\left(t_{n}\right)+\int_{t_{n}}^{t} F(s, x(s)) d s
$$

Lemma 1 is proved by straightforward verification.

Let $\overline{\mathbb{R}}=\mathbb{R} \cup\{ \pm \infty\}$. Let $I(\overline{\mathbb{R}})$ be the set of all intervals and $I(\mathbb{R})$ the set of all bounded intervals. For $\Omega \subset \overline{\mathbb{R}}$ we denote

$$
\mathbb{A}_{\Omega}=\{f: \Omega \rightarrow I(\mathbb{R})\}, \quad A_{\Omega}=\{f: \Omega \rightarrow \mathbb{R}\}
$$

Definition 2. [5] The low and upper boundary of Baire of $f \in \mathbb{A}_{\Omega}$ in the point $x$ we call the number

$$
I(f ; x)=\lim _{\delta \rightarrow 0_{+}} I(\delta, f ; x) \text { and } S(f ; x)=\lim _{\delta \rightarrow 0_{+}} S(\delta, f ; x)
$$

respectively, where

$$
\begin{aligned}
& I(\delta, f ; x)=\inf \{y: y \in f(t), t \in[x-\delta, x+\delta,] \cap \Omega\} \\
& S(\delta, f ; x)=\sup \{y: y \in f(t), t \in[x-\delta, x+\delta,] \cap \Omega\}
\end{aligned}
$$


By $\operatorname{gr}(h)$ we denote the graph of the function $h \in \mathbb{A}_{\Omega}$, i.e.

$$
\operatorname{gr}(h)=\{(x, y): x \in \Omega, y \in f(x)\} .
$$

Definition 3. [5] A completion of the graph of the function $f \in \mathbb{A}_{\Omega}$ we call the intervalued function $F: \mathbb{A}_{\Omega} \rightarrow \mathbb{A}_{\bar{\Omega}}$ defined by the formula

$$
F(f ; x)=[I(f ; x), S(f ; x)] .
$$

Let $\mathbb{F}_{\Omega}$ be the set of all intervalued functions which coindide with the completion of his graph, i.e.

$$
\mathbb{F}_{\Omega}=\left\{f \in \mathbb{A}_{\Omega}: F(f ; x)=f(x), x \in \Omega\right\}
$$

Let $f, g \in \mathbb{F}_{\Omega}$.

Definition 4. [5] The number

$$
h(f, g)=\sup _{(x, y) \in g r(f)} \inf _{(\xi, \eta) \in g r(g)} \max [|x-\xi|,|y-\eta|]
$$

is called one-sided Hausdorff metric.

We shall find approximations for the solution of the problem (1), (2), (4). For this aim we construct an ordinary differential equation, such that the solution of the two equations are nearly w.r. to the Hausdorff metric.

\section{Main Results}

We introduce the following condition:

$$
\Delta=\inf _{n}\left(t_{n+1}-t_{n}\right)>0
$$

Theorem 1. We assume that the impulse Cauchy problem (1), (2), (4) has for any $x_{0} \in \mathbb{R}$ a unique solution $x(t)$ and that the condition $(H 1)$ holds. Then for any $\varepsilon \in(0, \Delta)$ there exists a function $G_{\varepsilon}(t, y)(t \geq 0, y \in \mathbb{R})$ such that the Cauchy problem

$$
\begin{aligned}
\frac{d y}{d t} & =G_{\varepsilon}(t, y) \\
y\left(t_{0}\right) & =x_{0}
\end{aligned}
$$

has a unique solution $y(t)$ with $h(y, x)<\varepsilon$.

Proof. Let $\varepsilon>0$ be arbitrary choosen. We set

$$
G_{\varepsilon}(t, y)= \begin{cases}F(t, y)+\frac{1}{\varepsilon}\left(Q_{n}-I\right) x\left(t_{n}\right), & t \in\left(t_{n}, t_{n}+\varepsilon\right] \\ F(t, y), & t \in\left(t_{n}+\varepsilon, t_{n+1}\right]\end{cases}
$$


$n=0,1,2, \cdots, Q_{0}=I$.

The solution of the ordinary equation (6), (7) is given by the formula.

$$
y(t)=y\left(t_{n}\right)+\int_{t_{n}}^{t} G_{\varepsilon}(s, y(s)) d s
$$

It is not diffcult to check that for $n=1,2, \cdots$

$$
y\left(t_{n}\right)=x\left(t_{n}\right)
$$

holds. Indeed from (5), (8) and (9) it follows immediately that

$$
x\left(t_{1}\right)=y\left(t_{1}\right)
$$

We suppose that $y\left(t_{n-1}\right)=x\left(t_{n-1}\right)$ and we shall prove that $y\left(t_{n}\right)=x\left(t_{n}\right)$.

From (5), (8)and (9) it follows that

$$
x\left(t_{n}\right)=Q_{n-1} x\left(t_{n-1}\right)+\int_{t_{n-1}}^{t_{n}} F(s, x(s)) d s
$$

and

$$
\begin{aligned}
y\left(t_{n}\right)= & y\left(t_{n-1}\right)+\int_{t_{n-1}}^{t_{n}} G_{\varepsilon}(s, y(s)) d s \\
= & x\left(t_{n-1}\right)+\int_{t_{n-1}}^{t_{n-1}+\varepsilon} F(s, y(s)) d s+\int_{t_{n-1}}^{t_{n-1}+\varepsilon} \frac{1}{\varepsilon}\left(Q_{n-1} x\left(t_{n-1}\right)-x\left(t_{n-1}\right)\right) d s \\
& +\int_{t_{n-1}+\varepsilon}^{t_{n}} F(s, y(s)) d s \\
= & Q_{n-1} x\left(t_{n-1}\right)+\int_{t_{n-1}}^{t_{n}} F(s, x(s)) d s
\end{aligned}
$$

Hence

$$
y\left(t_{n}\right)=x\left(t_{n}\right)
$$

From (5), (8), (9) and (10) we obtain

$$
x\left(t_{n}+\varepsilon\right)=Q_{n} x\left(t_{n}\right)+\int_{t_{n}}^{t_{n+\varepsilon}} F(s, x(s)) d s
$$

and

$$
\begin{aligned}
& y\left(t_{n}+\varepsilon\right)=y\left(t_{n}\right)+\int_{t_{n}}^{t_{n+\varepsilon}} G_{\varepsilon}(s, y(s)) d s \\
= & x\left(t_{n}\right)+\int_{t_{n}}^{t_{n+\varepsilon}} F(s, y(s)) d s+\int_{t_{n}}^{t_{n+\varepsilon}} \frac{1}{\varepsilon}\left(Q_{n} x\left(t_{n}\right)-x\left(t_{n}\right)\right) d s \\
= & Q_{n} x\left(t_{n}\right)+\int_{t_{n}}^{t_{n}+\varepsilon} F(s, x(s)) d s
\end{aligned}
$$


Hence

$$
y\left(t_{n}+\varepsilon\right)=x\left(t_{n}+\varepsilon\right)
$$

From (8), (10) and (11) we obtain

$$
y(t)=x(t), \quad t \in\left[t_{n}+\varepsilon, t_{n+1}\right]
$$

and therefore $h(y, x)<\varepsilon$.

Remark 1. The function $G_{\varepsilon}(t, y)$ can be defined on the following way too

$$
G_{\varepsilon}(t, y)= \begin{cases}F(t, y)+P_{n}(t), & t \in\left(t_{n}, t_{n}+\varepsilon\right] \\ F(t, y), & t \in\left(t_{n}+\varepsilon, t_{n+1}\right]\end{cases}
$$

for $n=0,1,2, \cdots$, where $P_{0}(t) \equiv 0$,

$$
P_{n}(t)= \begin{cases}a_{n}\left(t-t_{n}\right)\left(t-\left(t_{n}+\varepsilon\right)\right), & t \in\left(t_{n}, t_{n+\varepsilon}\right) \\ 0, & t \notin\left(t_{n}, t_{n+\varepsilon}\right)\end{cases}
$$

for $n=1,2, \cdots$ and the numbers $a_{n}$ is given by

$$
a_{n}=\left(Q_{n} x\left(t_{n}\right)-x\left(t_{n}\right)\right)\left(\int_{t_{n}}^{t_{n}+\varepsilon}\left(t-t_{n}\right)\left(t-\left(t_{n+\varepsilon}\right)\right) d t\right)^{-1}
$$

Theorem 2. Let the following conditions are fulfilled:

1. The impulse Cauchy problem (1), (2), (4) has a unique solution $x(t)$ for any $x_{0} \in \mathbb{R}$.

2. The condition $(H 1)$ holds.

Then for any sufficiently small $\varepsilon>0$ there exists a function $G_{\varepsilon}(t, y),(t \geq 0, y \in \mathbb{R})$ such that the Cauchy problem (6), (7) has a unique solution $y(t)$ so that

$$
\|y-x\|_{p}=\left(\int_{0}^{\infty}|y(t)-x(t)|^{p} d t\right)^{\frac{1}{p}} \leq \varepsilon \quad(1 \leq p<\infty) .
$$

Proof. Let $\varepsilon>0$ be so small that

$$
\varepsilon_{n}=\frac{\varepsilon^{p}}{\left|M_{n}+1\right|^{p}}\left(\frac{p}{p+1}\right)^{n-1} \leq \Delta \quad(n=1,2 \cdots) .
$$

Here $M_{n}:=Q x\left(t_{n}\right)-x\left(t_{n}\right)$.

We set

$$
G_{\varepsilon}(t, y)= \begin{cases}F(t, y)+\frac{1}{\varepsilon_{n}}\left(Q_{n}-I\right) x\left(t_{n}\right), & t \in\left(t_{n}, t_{n}+\varepsilon_{n}\right] \\ F(t, y), & t \in\left(t_{n}+\varepsilon_{n}, t_{n+1}\right]\end{cases}
$$


$n=0,1,2, \cdots, Q_{0}=I$.

Let $y(t)$ be the solution of the problem (6), (7) and $x(t)$ the solution of the impulse problem $(1),(2),(4)$. Then for $t \in\left(t_{n}, t_{n}+\varepsilon_{n}\right)$ we have

$$
y(t)-x(t)=\left(Q_{n} x\left(t_{n}\right)-x\left(t_{n}\right)\right) \frac{t-\left(t_{n}+\varepsilon_{n}\right)}{\varepsilon_{n}}
$$

Because $y(t)=x(t),\left(t \in\left[t_{n}+\varepsilon_{n}, t_{n+1}\right]\right)$ we have

$$
\begin{aligned}
\|y-x\|_{p}^{p} & =\int_{0}^{\infty}|y(t)-x(t)|^{p} d t=\sum_{n=1}^{\infty} \int_{t_{n}}^{t_{n}+\varepsilon_{n}}\left|\left(Q_{n} x\left(t_{n}\right)-x\left(t_{n}\right)\right) \frac{t-\left(t_{n}+\varepsilon_{n}\right)}{\varepsilon_{n}}\right|^{p} d t \\
& \leq \sum_{n=1}^{\infty} \frac{\left|Q_{n} x\left(t_{n}\right)-x\left(t_{n}\right)\right|^{p}}{\varepsilon_{n}^{p}} \int_{t_{n}}^{t_{n}+\varepsilon_{n}}\left(t_{n}+\varepsilon_{n}-t\right)^{p} d t \leq \sum_{n=1}^{\infty} \frac{\left|M_{n}\right|^{p}}{\varepsilon_{n}^{p}} \frac{\varepsilon_{n}^{p+1}}{p+1}=\varepsilon^{p}
\end{aligned}
$$

i.e. $\|x-y\|_{p}<\varepsilon$.

Remark 2. The function $G_{\varepsilon}(t, y)$ can be defined by the following way too.

$$
G_{\varepsilon}(t, y)= \begin{cases}F(t, y)+P_{n}(t), & t \in\left(t_{n}, t_{n}+\varepsilon_{n}\right] \\ F(t, y), & t \in\left(t_{n}+\varepsilon_{n}, t_{n+1}\right]\end{cases}
$$

for $n=0,1,2, \cdots$, where $P_{0}(t) \equiv 0$,

$$
P_{n}(t)= \begin{cases}a_{n}\left(t-t_{n}\right)\left(t-\left(t_{n}+\varepsilon_{n}\right)\right), & t \in\left(t_{n}, t_{n}+\varepsilon_{n}\right) \\ 0, & t \notin\left(t_{n}, t_{n}+\varepsilon_{n}\right)\end{cases}
$$

for $n=1,2, \cdots$ and the number $a_{n}$ is given by

i.e.

$$
a_{n}=\left(Q_{n} x\left(t_{n}\right)-x\left(t_{n}\right)\right)\left(\int_{t_{n}}^{t_{n}+\varepsilon_{n}}\left(t-t_{n}\right)\left(t-\left(t_{n}+\varepsilon_{n}\right)\right) d t\right)^{-1},
$$

$$
a_{n}=-\frac{6 M_{n}}{\varepsilon_{n}^{3}} .
$$

Let $\varepsilon>0$ is so small that

$$
\varepsilon_{n}=\left(\frac{2}{3}\right)^{p} \frac{\varepsilon^{p}}{\left|M_{n}+1\right|^{p}}\left(\frac{p}{p+1}\right)^{n-1} \leq \Delta \quad(n=1,2, \cdots) .
$$

Then for $t \in\left(t_{n}, t_{n}+\varepsilon_{n}\right)$ we have

$$
\begin{aligned}
y(t)-x(t) & =x\left(t_{n}\right)-Q_{n} x\left(t_{n}\right)+\int_{t_{n}}^{t} P_{n}(s) d s=\int_{t_{n}+\varepsilon_{n}}^{t} P_{n}(s) d s \\
|y(t)-x(t)| & \leq \int_{t}^{t_{n}+\varepsilon_{n}}\left|P_{n}(s)\right| d s \leq \max _{t}\left|P_{n}(t)\right|\left(t_{n}+\varepsilon_{n}-t\right) \\
& =\left|P_{n}\left(t_{n}+\frac{\varepsilon_{n}}{2}\right)\right|\left(t_{n}+\varepsilon_{n}-t\right)=\frac{6\left|M_{n}\right|}{\varepsilon_{n}^{3}} \frac{\varepsilon_{n}^{2}}{4}\left(t_{n}+\varepsilon_{n}-t\right)=\frac{3\left|M_{n}\right|}{2 \varepsilon_{n}}\left(t_{n}+\varepsilon_{n}-t\right)
\end{aligned}
$$


Hence

$$
\begin{aligned}
\|y-x\|_{p}^{p} & =\int_{0}^{\infty}|y(t)-x(t)|^{p} d t \\
& \leq \sum_{n=1}^{\infty} \int_{t_{n}}^{t_{n}+\varepsilon_{n}}\left(\frac{3}{2}\right)^{p} \frac{\left|M_{n}\right|^{p}}{\varepsilon_{n}^{p}}\left(t_{n}+\varepsilon_{n}-t\right)^{p} d t=\left(\frac{3}{2}\right)^{p} \sum_{n=1}^{\infty}\left|M_{n}\right|^{p} \frac{\varepsilon_{n}}{p+1}=\varepsilon^{p}
\end{aligned}
$$

i.e. $\|y-x\|_{p}<\varepsilon$.

\section{References}

[1] D. D. Bainov and S. I. Kostadinov, Abstract impulsive differential equations, SCT Publishing, (1994), pp.167.

[2] D. D. Bainov and S. I. Kostadinov, Dichotomies and integral manifolds of impulsive differential equations, SCT Publishing, (1994), pp.110.

[3] V. Lakshmikantham, D. D. Bainov and P. S. Simeonov, Theory of impulsive differential equations, World Scientific, (1989), pp.273.

[4] A. M. Samoilenko and N. A. Perestyik, Differential equations with impulse effect, kiev, Visha Skola, (1987), pp.285.

[5] B. Sendov, Hausdorff approximations, Sofia, (1979), pp.370, (in Russian).

Faculty of Mathematics and Informatics, University of Plovdiv, Paissi Hilendarski, 4000 Plovdiv, Bulgaria. 